Journal of

Education and Practice

(JEP)

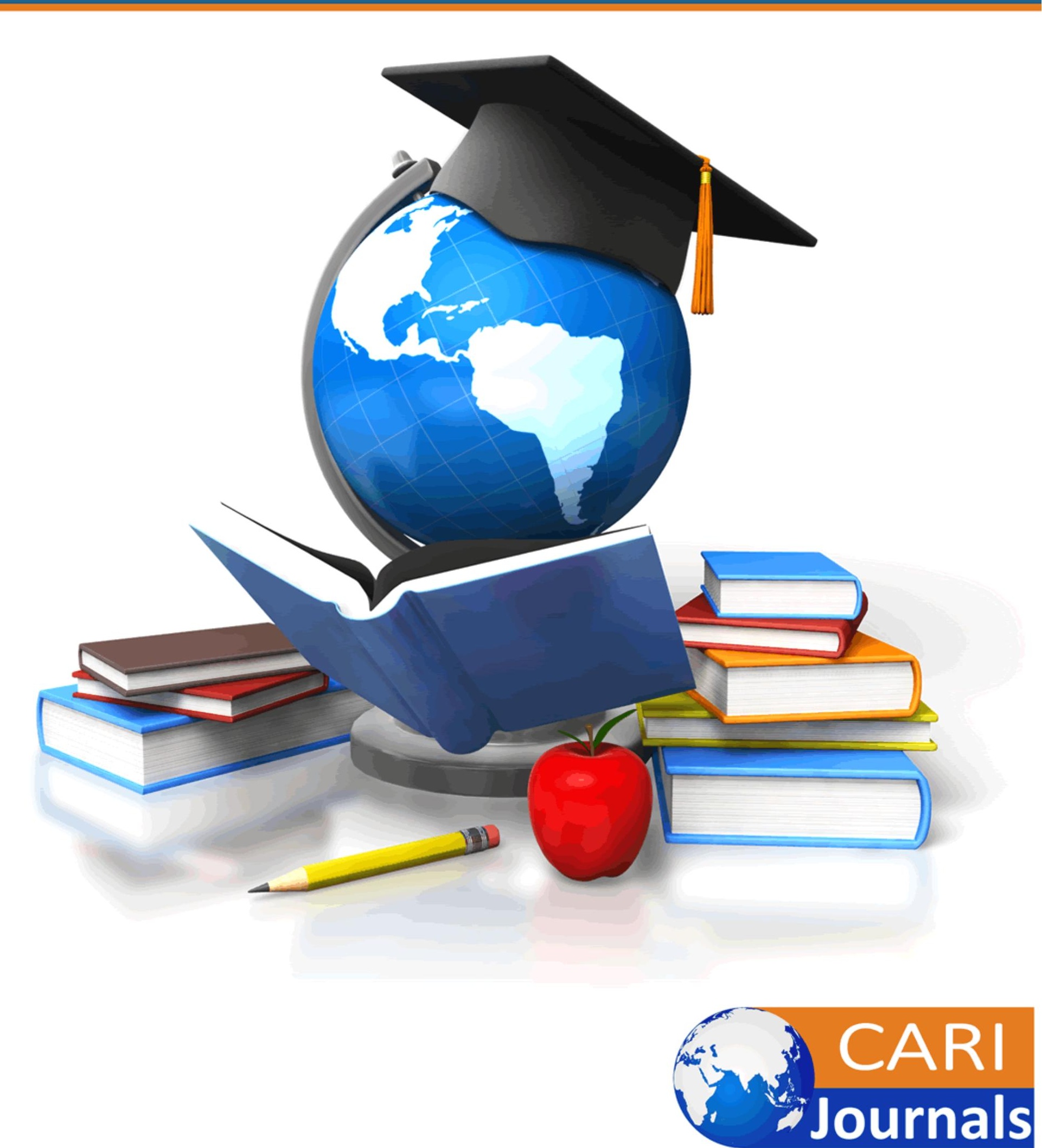




\title{
LA PROBLEMATIQUE DE L'ETHIQUE DANS LE CHAMP DES ENSEIGNANTS-CHERCHEURS : ENTRE LUTTES ET LOGIQUES TRANSACTIONNELLES. ETUDE A PARTIR DES ENSEIGNANTS CHERCHEURS DES UNIVERSITES D'ETAT DU CAMEROUN.
}

\author{
${ }^{1 *}$ Bienvenue Merci MOUDJOURI \\ Corresponding Author Email : moudjourib@gmail.com \\ Doctorant en Science politique, Université de Ngaoundéré, Cameroun.

\section{Resume`}

Objet: Les enseignants de l'enseignement supérieur exercent leur profession dans un cadre éthique qui leur est imposé comme valeur dans l'exercice des missions d'enseignement, de recherche et d'appui au développement. Ces différentes missions contiennent des prescriptions qui encadrent la profession. Cependant, l'éthique du corps enseignant ne fait véritablement pas l'objet de politiques publiques. Le corps enseignant est complexe par sa structuration et par les diverses trajectoires d'ascension professionnelle. Ces déterminants sont sources de luttes de classe et de lutte idéologique qui affectent l'éthique des enseignants. Les enseignantschercheurs entretiennent des relations horizontales et verticales qui sont conflictuelles et remettent en cause l'éthique. Les différentes luttes au sein du corps enseignant sont des luttes transactionnelles qui amenuisent l'éthique. Le corps des conférenciers est complexe de par sa structure et la diversité de ses parcours professionnels. Ces déterminants sont à l'origine des différentes luttes au sein des classes et des idéologies qui affectent l'éthique des conférenciers. Les professeurs entretiennent des relations horizontales et verticales conflictuelles qui rendent cette éthique sceptique.

Méthodologie: nous utilisons le constructivisme structurel pour expliquer la lutte entre le maître de conférence et l'institutionnalisme historique pour montrer comment l'université les intègre à la mission décrite par les pouvoirs publics

Trouver: Les différents combats au sein du corps enseignant sont des combats transactionnels qui affaiblissent cette éthique.

Une contribution unique à la théorie, à la pratique et à la politique: Ce sujet contribue à faire passer l'éthique dans le domaine de l'enseignement au-dessus de l'éthique classique

Mots clés: université, maître de conférences-chercheur, éthique, lutte transactionnelle, pouvoir

\section{INTRODUCTION}

La problématique de l'éthique s'impose dans les champs politiques, médiatiques, dans les corps de métier pour rendre acceptable ou humaniser les actions des différents acteurs dans chaque domaine spécifique.

Contexte

Dans l'enseignement supérieur, les institutions universitaires et les pouvoirs publics ont défini un code d'éthique tant pour la profession que pour les enseignants qui exercent l'activité d'enseignement du supérieur ${ }^{1}$. Elle tient généralement à la relation pédagogique de l'enseignant

\footnotetext{
${ }^{1}$ L'accent est mis par exemple sur l'éthique dans la recherche pour les multiples dérives enregistrées notamment le plagiat, l'attribution frauduleuse d'une production scientifique, etc.
} 
avec l'apprenant ${ }^{2}$ qui appelle à la responsabilité de l'enseignant ${ }^{3}$. Pourtant, les relations dans la profession enseignante ne sont pas uniquement les relations enseignant-étudiant. On en relève la relation horizontale ${ }^{4}$ et la relation verticale ${ }^{5}$. Ces relations sont pour la plupart encadrées. En contexte camerounais, l'éthique s'intègre dans les corpus d'évaluation pour l'ascension professionnelle ${ }^{6}$.

Au sein du corps enseignant, il y a une pathologie qui faiblement est prise en compte par les pouvoirs publics comme un problème et dont il faut y prendre des décisions pour la juguler. Il s'agit des luttes des enseignants. Ces luttes en contexte camerounais sont liées à la multitude des trajectoires d'ascension professionnelle. En effet, les pouvoirs publics ont mis en œuvre dans la politique d'ascension académique plusieurs trajectoires notamment celle de la trajectoire par le système national à travers le Commission Consultative des Institutions Universitaires (CCIU) et celle de la coopération internationale. La trajectoire à travers la coopération internationale comprend la trajectoire du Système CAMES, celle des pays coopérants notamment le système d'Agrégation française et le système de l'Habilitation des universités suisses et allemandes.

\section{Problème de recherche}

Cette diversité des trajectoires fait éclore les luttes idéologiques dans le corps enseignant spécifiquement pour les disciplines à agrégation. La complexité de ces luttes a une incidence sur les rapports entre les enseignants. La problématique qui guide notre travail procède de plusieurs questions: Quels sont les enjeux stratégiques de ces luttes et quelles sont les incidences de ces rapports conflictuels sur l'éthique ? Ces questions méritent une attention particulière car l'éthique les luttes des enseignants ne sont pas neutres, elles sont construites pour les enjeux, elles affectent à certains égards les comportements des enseignants.

\section{Finalités des objectifs}

Nous émettons l'hypothèse selon laquelle les logiques transactionnelles sont les déterminants de la construction des luttes qui flétrissent l'éthique des enseignants. Ce qui permet de comprendre les tenants et les aboutissants des luttes, de comprendre les stratégies entreprises par les enseignants-chercheurs pour accéder aux profits spécifiques qui sont en jeu dans le champ universitaire.

Deux cadres d'analyse sont recommandés pour l'analyse de notre sujet. Il s'agit du constructivisme structuraliste de $\mathrm{P}$. Bourdieu et de l'institutionnalisme historique. L'appréhension du corps enseignant sous l'angle d'un champ nous conduit à utiliser le constructivisme structuraliste de P. Bourdieu. La démarche constructiviste structuraliste est utile ici pour analyser le corps enseignant dans le champ de lutte pour l'accès à des postes de pouvoir au sein de l'institution. Tout de même, l'institutionnalisme historique est une autre

\footnotetext{
${ }^{2}$ J.Biron, « Pour une éthique partagée dans la profession enseignante », COFPE, mars 2014, disponible en ligne à l'adresse www.cofpe.gouv.qc.ca.

${ }^{3}$ J. Biron, « Pour une éthique partagée dans la profession enseignante », op cit.

${ }^{4}$ c'est à dire enseignant-enseignant
}

${ }^{5} \mathrm{La}$ relation verticale ici comprend la relation administration-enseignant, relation institutionenseignant, relation ministère-enseignant et relation pouvoir politique-enseignant

${ }^{6}$ L'éthique est l'un des référents de l'évaluation pour l'inscription dans les listes d'aptitude aux différentes
fonctions dans les différents arrêtés pour ladite inscription depuis l'arrêté n ${ }^{\circ} 0004 / \mathrm{MINESUP/CAB} \mathrm{du} 19$ janvier
1998 fixant les critères de recrutement et de promotion des personnels enseignants des Institutions Universitaires. 
méthode dont nous faisons recours. Elle permet de comprendre comment l'institution universitaire intègre le corps enseignant

Nous avons choisi comme échantillon le personnel enseignant des facultés des sciences juridiques et politiques de sept universités publiques ${ }^{7}$. En effet, il serait lourd d'étudier le personnel enseignant de toutes les chapelles pluridisciplinaires qui constituent les universités camerounaises. Les enseignants ciblés, constituent, à l'exception près, un terrain fertile pour l'observation, l'analyse du vécu quotidien des universitaires dans leur environnement interne. Cependant, il y a lieu de relever que les déterminants n'agissent pas de la même façon: les conditions de promotion académiques diffèrent parfois selon les disciplines ${ }^{8}$. Ce qui guide notre choix sur les enseignants qui connaissent une pluralité de choix dans les modes d'ascension professionnelle.

L'exploitation des données documentaires et de l'observation critique du corps enseignant permet de démontrer la construction des luttes des enseignants-chercheurs (1), ce qui conforte l'analyse des logiques transactionnelles contre l'éthique (2).

\section{I - LA CONSTRUCTION DES LUTTES DES ENSEIGNANTS-CHERCHEURS.}

Le champ des universitaires, dans la théorie des champs est un champ de lutte qui se situe à plusieurs niveaux. Les luttes sont un phénomène social construit par les enseignants-chercheurs. Ce qui fait du corps enseignant un champ de lutte idéologique qui amenuise l'éthique (1). Elle produit un effet sur le comportement réel des enseignants contraire à l'éthique (2).

\section{1 - Le corps enseignant, un champ de lutte}

Les enseignants des disciplines des sciences juridiques et politiques sont permanemment en situation conflictuelle dont le facteur déterminant est la pluralité des trajectoires d'ascension professionnelle. Ces trajectoires divergent par les titres qu'elles confèrent. Ce qui conduit à la septicémie de l'éthique par les luttes idéologiques d'une part (1) et à la lutte déloyale pour l'ascension professionnelle (2).

\section{I.1.1 - Les luttes idéologiques des enseignants}

La lutte idéologique dans le champ des universitaires se déroule entre les enseignants agrégés et les enseignants non agrégés. Cette lutte repose sur la tendance à la domination des agrégés sur les non agrégés car les pouvoirs publics ont fait des aménagements favorables aux enseignants agrégés. Il se crée une configuration des enseignants de rang magistral qui stimule un clivage entre les enseignants de rang magistral. Ce clivage est le fleuron de lutte idéologique des enseignants agrégés et des enseignants non agrégés.

La domination est donc au fondement de la lutte entre ces différents acteurs qui ont pour dénominateur commun le rang magistral mais dont les capitaux issus des différents titres conduisent à construire des frontières au sein du champ magistral. Cette lutte fait perdre le sens de l'éthique chez les enseignants qui sont pourtant tous des enseignants de rang magistral. Les

\footnotetext{
${ }^{7}$ L'université de Yaoundé depuis sa fission en 1993 n'offre plus de formation dans les filières des sciences juridiques et politiques, et de sciences économiques et de gestion qui sont l'apanage désormais de l'Université de Yaoundé II. On a donc la Faculté des Sciences juridiques et politiques de l'université de Yaoundé II, de l'Université de Ngaoundéré, de l'Université de Douala, de l'Université de Dschang, de l'Université de Maroua, de l'University of Buéa et enfin l'University of Bamenda.

${ }^{8}$ A titre d'illustration, les scientifiques ne progressent que par la voie des travaux dite la voie longue alors que dans les disciplines de droit et de science politiques connaissent une double progression selon les choix des enseignants.
} 
chapelles finissent par créer des clivages au lieu de la collaboration pour l'action collective. Si la domination est une hypothèse d'explication des enjeux de lutte idéologique ${ }^{9}$, il faut dire qu'elle est plus expressive avec les représentations dont s'en font les enseignants agrégés.

Les enseignants agrégés usent des symboles pour exprimer la différence avec les autres enseignants. Le trophée du concours d'agrégation est le titre d'enseignant agrégé, que les titulaires exhibent à la communauté universitaire. Le trophée du concours s'exprime dans leur cachet (nominatif) où les enseignants agrégés précisent toujours leur trajectoire du CAMES : Agrégé de (...) ou Agrégé CAMES en (...). Pourtant, cette agrégation leur donne le grade de Maître de Conférences au même titre que les enseignants qui sont passés par l'évaluation du système national. D'ailleurs, ils sont les Maîtres de Conférences agrégés. Cette exhibition du trophée de l'agrégation pose également un problème éthique.

Les représentations du trophée participent dans le jeu de lutte idéologique dont l'objectif est le déclassement des enseignants de rang magistral issu du système national. Ce déclassement des enseignants non agrégés n'est pas éthique, l'agrégation n'est pas une fin en soi, mais un moyen d'ascension professionnelle au même titre que le CCIU.

Les enseignants non agrégés fustigent la considération selon laquelle les enseignants agrégés sont supérieurs à eux sur le seul fait qu'ils ont réussi au concours d'Agrégation. Pour eux, l'Agrégation n'est pas un espace de mesure de la connaissance ${ }^{10}$. Les enseignants agrégés se comportent comme si le CAMES est un instrument infaillible d'évaluation pourtant de nombreuses imperfections entachent la procédure d'évaluation. D'ailleurs, ce CAMES est ébranlé par des questions de corruption, de magouille, de fraude des membres du jury pour faire passer des candidats. C'est alors que certains responsables des sections d'évaluations ont pris des sanctions ${ }^{11}$.

L'éthique s'introduit également avec l'approche selon laquelle l'agrégation confère la reconnaissance internationale. Il est un construit sans base solide, pour des intérêts de leurs titulaires. La reconnaissance internationale se situe dans les productions scientifiques dans les revues internationales, dans les colloques et symposiums internationaux, dans les invitations dans les centres de recherche de référence. Ce sont les déterminants que les enseignants non agrégés possèdent et qui les font s'affirmer sur la scène scientifique.

\section{I.1.2 -Les luttes déloyales d'ascension professionnelle}

L'éthique est mise à épreuve par un autre phénomène pathologique au sein du champ enseignant-chercheur. Il s'agit des stratégies des enseignants pour freiner l'ascension professionnelle. Pendant que les enseignants luttent pour changer de grade d'autres enseignants s'érigent en écluse pour freiner leur projet. C'est ce que nous appelons ici les luttes déloyales ; elles sont une pathologie qui affecte voire ternie la dimension éthique.

${ }^{9}$ Ibid.

\footnotetext{
${ }^{10}$ Entretien du 23 novembre 2018 à Yaoundé avec un Professeur titulaire .

${ }^{11}$ C'est le cas du Conseil africain et malgache qui a sanctionné des universitaires lors de sa $36^{\text {ème }}$ session tenue à Cotonou du 27 au 30 mai 2019 six professeurs d'Université dont les sanctions allaient de la suspension au programme du Cames et à l'annulation du grade. Pour des raisons d'éthique nous n'allons pas faire mention de leurs noms dans ce travail, ils sont consultables dans sur internet.
} 
Les canaux d'ascension professionnelle sont faits de critères objectifs ${ }^{12}$ auxquels certains critères subjectifs interviennent du fait du jeu des acteurs impliqués dans le processus d'évaluation. Ces critères subjectifs impactent le processus d'évaluation quelle que soit la voie empruntée, ils apparaissent comme un fonctionnement antinomique.

Les enseignants vont développer des stratégies pour retarder le changement de grade des autres enseignants qui aspirent accéder à la classe supérieur. Ces stratégies sont entre autre le retard dans la signature des rapports par le chef de département et le doyen, la production des rapports négatifs sur les enseignants en dépit des rapports favorables du conseil d'établissement signé par le doyen et de l'Assemblée du département.

Le changement de grade constitue un moment de règlement de compte à certains enseignants. Il arrive que le Chef de département qui doit signer la liste des rapports, mémoires et thèses dirigés ou codirigés ${ }^{13}$ par un Chargé Cours ou un Maître de Conférences s'absente jusqu'à la date limite de recevabilité des dossiers ${ }^{14}$. Cette technique est aussi employée par le Chef de l'établissement. Ces stratégies, qui n'ont pour objectif que de retarder l'avancement d'un enseignant, ne sont pas éthiques.

Le moins que l'on puisse dire, c'est que l'éthique dans le champ des universitaires est au rabais au regard des luttes idéologiques pour discréditer les enseignants non agrégés et au regard des stratégies développées pour retarder l'ascension professionnelles des enseignants. Ces actions ont une influence sur le comportement dans le réel des enseignants.

\section{I.2 - Le comportement dans le réel des enseignants}

Dans le système universitaire, les enseignants entretiennent des relations de domination et de subordination qui sclérosent l'éthique. Le comportement des enseignants dans le réel est teinté des luttes horizontales (2) et des luttes verticales (1).

\section{I.2.1 - Les luttes verticales des enseignants}

Le comportement vertical est le comportement des enseignants à l'égard de la hiérarchie de la faculté que sont le doyen et les chefs de département. A ce comportement vertical correspondent les luttes verticales. Les enseignants-chercheurs ont des relations de collaboration et des relations conflictuelles avec leur hiérarchie. Les enseignants sont soumis à la hiérarchie pour l'acquisition des enseignements. Cette soumission à la hiérarchie pour l'acquisition d'un enseignement n'est éthique, c'est une obligation pour l'enseignant d'enseigner puisqu'il a été expressément recruté pour l'enseignement.

La longévité aux postes stratégiques va contribuer à la personnification dudit poste de telle sorte que les enseignants vont être soumis à la domination du doyen par exemple. Ils subissent des frustrations lorsque le doyen leur rappelle tout le temps que c'est lui le doyen, ce qui donne l'impression de la personnification du poste, d'une patrimonialisation de la fonction de doyen. Cette personnification n'est pas éthique car il n'est de poste acquis de façon héréditaire, il n'est pas une fonction patrimoniale, familiale. L'éthique voudrait que les fonctions administratives soient exercées de façon désintéressée, elle appelle au sens la dépersonnalisation des fonctions, à un civisme professionnel et administratif.

\footnotetext{
${ }^{12}$ Ces critères objectifs sont contenus dans les différents arrêtés portant conditions d'inscription sur les listes d'aptitude aux fonctions de Chargé de Cours, Maître de Conférences et Professeur.

${ }^{13}$ Ibid.

${ }^{14}$ Entretien du 2 décembre 2018 avec un Professeur à Yaoundé.
} 
Les enseignants se livrent des luttes pour la direction des travaux qui leur permettraient d'avoir le nombre requis d'étudiants à encadrer, ce qui les permet de postuler pour l'inscription sur les listes d'aptitude aux fonctions de Maîtres de Conférences ou de Professeurs. C'est ainsi que pour contrecarrer un enseignant, les autorités retirent la direction d'un mémoire à un enseignant, ce qui limite logiquement sa capacité à satisfaire au critère de la direction des mémoires. Cet acte n'est également pas éthique, il ne reflète pas une gestion professionnelle des ressources humaines.

\section{I.2.2 - Les luttes horizontales des enseignants}

Les enseignants entretiennent un autre type de relation, celle qui se déroule entre eux que nous qualifions decomportement horizontal. C'est ce comportement que les enseignants entretiennent entre eux du fait de l'appartenance au même corps, au-delà des grades et des fonctions. Les relations horizontales des enseignants sont tous aussi pacifiques que conflictuelles. Sous l'angle conflictuel, on relève des luttes horizontales. Il est difficile de faire des statistiques pour ressortir le type de relation qui dominent. Les luttes des enseignants ne sont pas seulement des luttes pour accéder à un grade supérieur, elles sont également des luttes d'affirmation de soi au sein du groupe, des luttes pour se maintenir dans le circuit du système.

Les tensions ouvertes avec les autres enseignants pour des raisons académiques et des raisons extra-académiques affectent les relations des enseignants. Pour ce qui est des tensions académiques, il s'agit des luttes pour les enseignements. Le retrait d'un enseignement à un enseignant éveillera les tensions entre lui et l'enseignant qui a obtenu cet enseignement à ses dépens. Il se forme une timidité, une froideur dans leur rapport et parfois on aboutit à une rupture de relations entre ces enseignants.

Les tensions ouvertes entre les enseignants ont parfois des raisons extra-académiques mais elles se répercutent à l'université. Les enseignants se livrent à des conflits internes qui prennent une ampleur inimaginée. Les enseignants, partis au conflit, font recours à leur hiérarchie au sein de la faculté ou font recours au recteur par des dénonciations des différends qu'ils ont avec leurs collègues. A ces espaces, s'ajoutent les amphithéâtres qui deviennent aussi des arènes d'expression de luttes des enseignants entre eux. La circulation des différends personnels des enseignants entre eux dans l'espace des étudiants pose un problème d'éthique dans la conservation et la dissimulation des crises de personnalité. Les enseignants n'agissent pas de façon responsable, ils sont mus par leur égo, leur passion, leur pulsion. Dans ces cas de figure, l'éthique disparait au profit des intérêts des enseignants.

Le moins que l'on puisse dire, c'est que les enseignants-chercheurs, loin de constituer le dernier rempart de la société sont en marge d'éthique. Les enseignants ont créé des clivages idéologiques, qui sont contraires à l'éthique. Ainsi, par leur comportement, l'éthique est mise à l'épreuve. Cette considération au rabais de l'éthique porte les germes des luttes transactionnelles.

\section{II - LES LOGIQUES TRANSACTIONNELLES DES DIFFERENTES LUTTES}

Les différentes luttes qui sont présentes au sein des enseignants-chercheurs entachent l'éthique. Les luttes idéologiques et les luttes de changement de grade poursuivent des logiques transactionnelles qui présentent un même caractère à savoir l'occupation des espaces de pouvoir (1). Les enseignants circulent dans l'espace public qui, en soit, est truffé de dérèglement éthique (2).

\section{II.1 - Les luttes d'occupation des espaces de pouvoir}


Les luttes au sein du corps enseignant ne sont pas neutres. Elles poursuivent des enjeux stratégiques qui se déchiffrent en postes stratégiques au sein de l'université (1) et en dehors d'elle (2).

\section{II.1.1 - Les luttes d'occupation des postes stratégiques à l'université}

Dans les universités d'Etat, les postes administratifs sont convoités par les enseignants, qui doivent accéder à un certain grade pour espérer les occuper. Les occupations de ces postes font intervenir les paramètres formels et informels. L'intervention des paramètres formels conduit à la fragilisation de l'éthique. Au sein de l'université, les postes stratégiques sont les postes de recteurs, de doyens, de chefs de département. Ce sont des postes par essence de décision ${ }^{15}$.

Les enseignants au rang magistral sont en attente d'un arrêté ou d'un décret ${ }^{16}$ dès l'instant où le CCIU décide de leur inscription sur les listes d'aptitude aux grades de Maître de Conférences ou de Professeurs. Tout se passe comme si accéder au rang magistral signifie accéder à un poste, il devient un concours d'occupation de poste dont la condition est celle d'être de rang magistral. Pourtant, c'est l'espace dans lequel les enseignants doivent justifier de leur qualité d'enseignant de rang magistral par les travaux de qualité.

Les enseignants-chercheurs miroitent l'occupation des postes au sein des facultés dont la condition semble être reconnue comme un enseignant de rang magistral. En fin de compte, l'on s'aperçoit que l'enjeu de lutte des enseignants, c'est l'occupation des postes stratégiques au sein des facultés. La quête ou l'attente d'un décret devient une obsession lorsque l'enseignant est au rang magistral. Il rompt avec l'éthique par des pratiques perverses qui le portent à un poste stratégique.

\section{II.1.2 - Les luttes d'occupation des postes de pouvoir en dehors de l'université}

Les espaces de pouvoir se trouvent au sein de l'université et dans l'espace public notamment dans le champ gouvernementale. Le pouvoir politique a monopolisé et réduit la dévolution du pouvoir dans la haute fonction administrative par le décret. L'espace public regorge des avantages matériels et immatériels. La quête des postes interroge sur les dispositions éthiques des enseignants car leur mission reste grandement à explorer pour contribuer au développement.

La nomination dans l'appareil gouvernemental procède de la compétence scientifique des enseignants et de la proximité idéologique qui est plus décisive ${ }^{17}$. L'enseignement supérieur devient comme un espace de recrutement dans la fonction gouvernementale. Il devient un autre enjeu de lutte des enseignants qui ambitionnent se reconvertir dans ce champ comme les fonctionnaires.

Les enseignants sont dans la quête explicite des postes dans l'appareil gouvernemental. Cette quête des postes met au rabais l'éthique des enseignants qui ne font pas de leur profession une fonction convoitée pour le capital culturel dont ils disposent. Au plafond de verre, c'est-à-dire

\footnotetext{
${ }^{15}$ Les postes de vices recteurs, de Secrétaire général de l'université, de vice-doyen sont aussi importants, mais ils ne sont pas des postes ayant le même pouvoir que les titulaires des postes administratifs, ce sont des postes de décision. Le recteur par exemple à le pouvoir de recrutement des personnels d'appui alors que les autres vices recteurs et le Secrétaire Général ou le Conseiller technique auprès du recteur ne peuvent le faire. Le doyen pour sa part par exemple est un gestionnaire alors que ses vice-recteurs ne le sont.

${ }^{16} \mathrm{~J}$. Onana, «Etre savant et intellectuel au Cameroun», in A. KOUVOUAMA , (éd.), Figures croisées d'intellectuel, trajectoires, modes d'action, production, Paris, Karthala, p.253-256.

${ }^{17}$ F. Dreyfus, « Les hauts fonctionnaires et la politique », in A. COHEN et al., Nouvel manuel de Science politique, Paris, La Découverte, 2009.
} 
dans la classe de Professeur, les enseignants font le minimum de ce qui leur est demandé. Pourtant, c'est l'espace dans lequel les pouvoirs publics attendent plus des enseignants au regard des primes de recherche ${ }^{18}$ pour rehausser le niveau de l'enseignement supérieur.

Les espaces de pouvoir sont des espaces qui flétrissent l'éthique des enseignants, car les enseignants sont à la quête du décret qui leur permet d'accéder et d'exercer le pouvoir. Les enseignants ne mettent plus l'éthique au centre de leurs actions, de leur profession, ce qui importe c'est les transactions que comportent le corps enseignant, et spécifiquement le rang magistral. D'ailleurs, les enseignants ont tendance à délaisser la profession enseignante pour s'occuper d'autres sphères qui ne renforcent pas leur position dans le champ des universitaires.

\section{II.2 - Le sacrificiel de l'éthique au profit de la circulation dans l'espace public}

Les enseignants-chercheurs des universités d'Etat poursuivent leurs intérêts dans et à l'extérieur de l'université, dans des champs professionnels autres que le champ universitaire. La réussite sociale est le nœud commun qui les lie en dépit des stratégies développées pour y parvenir. C'est ainsi que l'on retrouve les enseignants dans les champs qui contribuent à leur fabrication sociale. Le recours à d'autres champs pour la fabrication des enseignants est-il éthique ? L'éthique des enseignants l'espace politique (1) se pose également au même titre que la question de l'éthique l'espace médiatique(2).

\section{II.2.1 - L'éthique des enseignants dans l'espace politique ?}

Les enseignants investissent le champ politique sous différentes catégories. Certains enseignants se constituent en professionnels de la politique ${ }^{19}$ par la conquête des postes électifs à travers lesquels ils entendent faire une activité professionnelle parallèlement à la profession enseignante du supérieur. Une autre catégorie d'enseignants est constituée en activiste politique. Ces enseignants s'intéressent à l'actualité politique et à la chose politique d'une part, et d'autre part ils contribuent à la compréhension et à la diffusion des idées politiques dans la société. L'intrusion dans le champ politique poursuit des sous-entendus qui se dessinent en termes de position dans l'appareil gouvernemental ou de l'occupation des postes administratifs à l'université.

Cet usage de la politique traduit la logique transactionnelle qui se joue au sein du corps enseignants et entre ces derniers et les acteurs politiques. L'usage de la politique au sein de la profession enseignante du supérieur soulève des interrogations sur l'éthique des enseignants. Ce recours à la politique va impulser une forte dépendance à la politique pour une activité publique déclarée apolitique par les pouvoirs publics ${ }^{20}$.

Les enseignants espèrent une ascension sociale en bénéficiant d'un texte qui va leur conférer du pouvoir dans une fonction gouvernementale. Dans cet ordre d'idées, les enseignants font de la politique pour se maintenir à leur poste, ils sont soumis voire contraints indirectement car le pouvoir politique a fait des postes administratifs des instruments de captation politique. La lutte des enseignants dans le champ politique est une lutte pour la conservation de leur poste. A certains égards, l'investissement dans le champ politique a pour objectif de gagner en position

\footnotetext{
${ }^{18}$ Décret n²009/121du 08 avril 2009 portant création, organisation et fonctionnement d'un compte d'affectation spéciale pour la modernisation de la recherche dans les universités d'Etat.

${ }^{19}$ J. Onana, Initiation à la Science Politique, Paris, Harmattan, 2009.

${ }^{20}$ M. Fouda Ndjodo et C. Awono Onana, «Les Réformes de la gouvernance dans l'enseignement supérieur camerounais », UNESCO 2012.
} 
dans l'administration de la faculté ou dans l'administration de l'université. On est ici en présence de la lutte subsistancielle qui est liée à l'engagement politique qui entache l'éthique.

$\mathrm{Au}$ regard de ces considérations, les enseignants recourent très peu à leur compétence scientifique pour accéder aux postes administratifs dans et en dehors de l'université. Ils utilisent le champ politique comme un canal d'échange de leur loyauté, de leur allégeance au pouvoir politique, ou aux dirigeants de la faculté pour obtenir des faveurs. L'enseignement se constitue en une faveur alors qu'il est une obligation professionnelle. Leur comportement suscite un regard critique sur l'éthique pour comprendre tout le marchandage qui se joue dans le corps. Il n'est pas d'éthique dans toutes ces transactions qui renferment les enseignants dans des pratiques non autorisées, ou encore qui les confinent dans des positions de faiblesse devant les dépositaires du pouvoir.

Les rapports au politique sont guidés fatalement par des débouchés, on ne s'imaginerait pas un enseignant qui ferait de la politique pour ne rien attendre en retour. D'ailleurs, les enseignants ont fait du champ politique un moyen pour parvenir à leur ambition. Une fois recruté comme personnel de l'enseignement supérieur dans une université, les enseignants aux ambitions de pouvoir ont tendance à se frayer un chemin dans le champ politique qui leur permettra d'atteindre leur objectif.

\section{II.2.2 - La question d'éthique des enseignants dans l'espace médiatique.}

L'espace médiatique est constitué des médias classiques notamment la radiodiffusion ${ }^{21}$, la télévision $^{22}$, la presse écrite ${ }^{23}$ et les nouveaux médias que sont les réseaux sociaux notamment Facebook, Twitter, et bien d'autres. Ces médias sont des espaces d'expression des acteurs qui ne sont plus seulement les professionnels des médias. Les enseignants-chercheurs sont les acteurs qui investissent les médias classiques comme consultants, comme conseillers ou invités. Aucune semaine ne se passe sans que l'on ait eu l'intervention d'un enseignant sur un média pour telle question ou pour telle autre.

L'irruption des enseignants-chercheurs dans les médias n'est pas une antinomie de la profession. Elle est même louable car les enseignants apportent du crédit à un média par les explications objectives qu'ils apportent sur une question qui suscite l'attention de l'opinion publique. Les enseignants estiment que leur irruption dans l'espace public est un engagement citoyen, un engagement pour l'action collective et dans la construction de la Nation par la socialisation politique. Les enseignants pensent que leur premier rôle dans l'espace public c'est d'édifier les citoyens sur le champ du pouvoir, ses diverses approches, ses formes, ses typologies, ses caractères ${ }^{24}$. Le champ du pouvoir est complexe et la masse des citoyens est mal socialisée, ils ne connaissent le pouvoir que sous sa forme superficielle, représenté par les dirigeants, et à l'occasion des luttes pour l'occupation des espaces de pouvoirs.

Cette approche est propre pour l'éthique, elle est la forme dont les pouvoirs publics, l'opinion publique et la communauté universitaire accueillent favorablement. Les enseignant-chercheurs dans ce cadre sont bien indiqués pour jouer ces rôles. Cependant, les rapports des enseignants

\footnotetext{
${ }^{21}$ Nous pouvons citer en ce qui concerne les plus suivis au Cameroun : Cameroon Radio and Television (CRTV), Canal 2 International, Vision 4, Equinoxe TV, STV2, LTM, etc.

${ }^{22}$ Nous avons la Radio nationale, Balafon, Radio Siantou, etc.

${ }^{23}$ Nous pouvons citer Cameroon Tribune, Le Messager, Emergence, Le Soir, L'œil du Sahel, le Jour, Mutations, The Guardian, The Post, etc.

${ }^{24}$ J. Onana, Initiation à la Science Politique, op cit.
} 
avec les médias ne sont pas toujours parfaits au point où l'on enregistre des dérives à l'éthique, ou encore on interroge l'éthique au regard de leur comportement dans les médias qui frisent avec leur position socioprofessionnelle.

La prégnance des enseignants dans les médias soulève la question de l'éthique à plus d'un titre. Lorsque les enseignants pensent que la place des universitaires est dans les laboratoires, dans les colloques, dans les conférences, les symposiums, dans les revues scientifiques, cela appelle à une responsabilisation dans la profession enseignante. C'est dans ces espaces que l'on fait la science, dans les médias on ne discute pas de science. Les enseignants subissent l'orientation des journalistes qui orientent les enseignants dans le sens qu'ils ont prévu à l'avance ou dans leur ligne éditoriale. Les enseignants qui passent plus de temps dans les médias se constituent en vedettes, alors que c'est dans les revues qu'on les attend. On parle dans ce cadre de la vedettisation des enseignants c'est-à-dire ce mécanisme qui consiste pour les enseignants à se constituer en vedette dans les médias.

L'éthique interpelle sur l'opportunité de l'intervention médiatique des enseignants, sur la qualité des membres du panel car les débats médiatiques très souvent ne sont pas pédagogiques au regard du comportement de certains panelistes. Ils sont de tous les ordres (avocat, économiste, journaliste, leader d'opinion, homme d'affaire, syndicaliste) ayant été socialisés dans leur corps de métier et ayant leur tempérament. Par conséquent, les interventions peuvent être contraires et contradictoires au point d'irriter la sensibilité certains panélistes.

Lors d'un débat télévisé sur une chaine de télévision privée un enseignant agrégé de Droit a été interpellé par un paneliste qui lui signifiait que si c'est ce qu'il enseigne aux étudiants ça ne vaut pas la peine. Cette interpellation a suscité de l'indignation au sein de la classe enseignante pour un panéliste qui fait du trafic d'influence du fait de sa position politico-administrative. Il est dans ce cadre peu indiqué pour un enseignant de discuter avec des individus qui ne comprennent pas la science. Ce qui interpelle sur l'éthique.

Par ailleurs, les enseignants se livrent à des luttes dans les médias pour la défense de leur chapelle et convictions politiques respectives. Ces luttes prennent des formes inattendues où les clivages sont très forts. Les enseignants se polarisent et se radicalisent dans des positions idéologiques qui deviennent des luttes entre enseignants ${ }^{25}$. Ils se comportent comme des individus appartenant à des corps distincts et se livrent à des injures dans les médias. On s'interroge sur la place qu'occupe l'éthique dans la vie ces enseignants dont la communauté universitaire a du respect. Dans ces luttes, ils perdent le respect qui les élevait au rang des maîtres de la science.

Par ailleurs, les enseignants choisissent le canal des réseaux sociaux pour se livrer à des luttes idéologiques ${ }^{26}$ quant à savoir qui est enseignant chercheur et qui ne l'est pas, pourquoi un enseignant devrait se consacrer à la recherche plutôt qu'aux débats médiatiques. Ces luttes ont envenimé Facebook au point où la communauté universitaire s'est impliquée pour atténuer les dérives et mettre fin au différend qui prenait une autre proportion. L'éthique est convoquée pour rendre compte de ce que les enseignants se livrent bataille en dehors du champ universitaire. Les enseignants perdent en crédibilité et en respect pour leur comportement qui aurait pu être

\footnotetext{
${ }^{25}$ L'élection présidentielle du 07 octobre 2018 sert de parfait exemple.

${ }^{26}$ Deux enseignants de Science Politique (dont nous tairons les noms) appartenant à la même université au Cameroun se sont livrés à des échanges virulents sur les réseaux sociaux indignant la communauté universitaires et les médias.
} 
évité ou pour le fait qu'ils auraient pu gérer leur différend en tenant compte de leur fonction socioprofessionnelle et de leur rôle dans la société.

Ces luttes politiques et académiques dans les médias et surtout dans les nouveaux réseaux sociaux ont terni l'image que représente un enseignant-chercheur. L'opinion publique en a fait une analyse de lutte de positionnement et la communauté universitaire en a perçu une lutte de leadership scientifique. Ces luttes, quels que soient leurs clivages ont exposé le corps enseignant dans son quotidien, dans le réel. L'éthique disparait pour faire place à des transactions entre protagonistes.

\section{CONCLUSION}

Il était pour nous question de vérifier l'hypothèse selon laquelle les logiques transactionnelles sont les déterminants de la construction des luttes qui discréditent l'éthique des enseignants. Pour cela, l'analyse nous a conduit à élaborer des schèmes de la mise à l'épreuve de l'éthique par les luttes des enseignants. Ces luttes ne sont pas seulement idéologiques, mais elles sont également des luttes de classe dans lesquelles les enseignants utilisent des stratégies de ralentissement ou de freinage des enseignants dans leur ambition d'ascension professionnelle. Ces luttes impactent sur l'éthique que l'on attribue aux enseignants comme modèle de la société. Par ailleurs, les comportements des enseignants dans le réel sont faits de pathologies tant dans leur relation au groupe que de leur relation à la hiérarchie de la faculté.

Les luttes des enseignants-chercheurs ne sont pas neutres, elles sont transactionnelles. Les enseignants-chercheurs utilisent leur position notamment leur titre et leur rang magistral pour la conquête des espaces de pouvoir qui se situent dans la faculté ou à l'université globalement, et dans l'appareil gouvernemental. Dans leur logique transactionnelle, les enseignants recourent à l'espace politique et à l'espace médiatique dans lesquels ils poursuivent leur lutte idéologique et leur lutte pour l'accès aux postes de pouvoir. Leurs intérêts priment sur l'éthique qui subit le choc des luttes politiques, de classe, et même des luttes scientifiques.

Les enseignants-chercheurs fragilisent l'éthique par leurs différentes luttes. Ces luttes ne sont pas inscrites dans les politiques du corps enseignants, pour le moins l'éthique est positive quand les luttes contribuent à l'ascension professionnelle des enseignants-chercheurs. Elle est négative quand les enseignants se livrent à des luttes pour occuper des espaces de pouvoir. Ici, les enseignants utilisent tous les moyens, et se donnent tous les coups ${ }^{27}$ pour y parvenir. Ces moyens et ces coups ne sont pas éthiques ou du moins ils fragilisent l'éthique que l'on reconnait aux personnels enseignants des universités.

\section{Recommandations}

Si les pouvoirs publics avaient défini un mode électoral d'accès aux postes administratifs, ces luttes devraient prendre une autre forme, l'éthique ne subirait pas les rapports de force des enseignants dans leur différentes trajectoires. L'on s'attendrait à une autre lutte. Si les pouvoirs publics ne s'étaient pas constitués en architecte de carrière professionnelle en laissant les enseignants dans leur corps et leur profession, l'éthique prendrait une autre forme, elle serait

\footnotetext{
${ }^{27}$ L. Ngwé, «Diplômes, grades et postes : bureaucraties universitaires, bureaucraties politiques et effets sur la dynamique du champ académique au Cameroun », in L. Ngwé et H.D.P. Pokam, L'enseignement supérieur depuis la réforme de 1993, CODESRIA, 2016.
} 
moins exposée aux différends des enseignants. L'éthique doit être reconstruite pour que les enseignants s'occupent effectivement des missions de l'université.

\section{Bibliographie :}

APHEC (2016), Code d'éthique des enseignants de l'HEC Montréal, Montréal.

BIRON J. (2014), «Pour une éthique partagée dans la profession enseignante», COFPE, , disponible en ligne à l'adresse www.cofpe.gouv.qc.ca.

BOURDIEU P.(1975), «La spécificité du champ scientifique et les conditions sociales $\mathrm{du}$ progrès de la raison », Sociologie et sociétés, vol 7 (1), p. 92, https:/doi.org/10.7202/00108ar, consulté le 06 février 2018.

BOURDIEU P. et WACQUANT L.J.D. (1992), Réponses... Pour une anthropologie réflexive, Paris, Seuil.

BRECHON P. (2006), Les Grands courants de la sociologie, Grenoble, PUG.

Dreyfus P. (2009), «Les hauts fonctionnaires et la politique», in COHEN A. et al. , Nouvel manuel de Science politique, Paris, La Découverte.

FOUDA NDJODO M. et AWONO ONANA C. (2012), «Les Réformes de la gouvernance dans l'enseignement supérieur camerounais », UNESCO.

GROFFIER E. (2014), Réflexions sur l'Université. Le devoir de vigilance, Québec, PUL, $200 \mathrm{p}$.

LABROUSSE A.( 2014), «Institutionnalisme et théorie de la régulation: quelle théorie ? Eléments de réflexion », sciencesconf.org :afep2014:33809, consulté le 03 février 2019.

MEI-CHIANG S.et al (2012), «Analyse de la réforme administrative taiwanaise du point de vue de l'institutionnalisme historique», Revue Internationale des Sciences Administratives, 2012/2, vol 78, p.323-345. Article disponible en ligne à l'adresse: http://www.cairn.info/revue-internationale-des-sciences-administratives-2012-2-page-323-

htm. Article consulté le 05-06-2012.

NGWE L. (2016), «Diplômes, grades et postes: bureaucraties universitaires, bureaucraties politiques et effets sur la dynamique du champ académique au Cameroun », in NGWE L.et POKAM H.D (2016), L'enseignement supérieur depuis la réforme de 1993, CODESRIA.

ONANA J. (2009), Initiation à la Science Politique, Paris, Harmattan.

ONANA J. (2008), «Être savant et intellectuel au Cameroun », in A. KOUVOUAMA (éd.), Figures croisées d'intellectuel, trajectoires, modes d'action, production, Paris, Karthala, p.253-256. 